\title{
Lignin Deposition In Arabidopsis thaliana Cell Walls Unveiled By Ptychographic X-Ray Computed Tomography (PXCT).
}

$\underline{\text { Carla Polo }^{1}}{ }$, Luciano Pereira ${ }^{2}$, Denisele Flores ${ }^{2}$, Manuel Guizar-Sicairos ${ }^{3}$, Mirko Holler ${ }^{3}$, Paulo Mazzafera $^{2}$, Juliana Mayer ${ }^{2}$, Harry Westfahl ${ }^{1}$, Florian Meneau ${ }^{1}$

1. Brazilian Light Synchrotron Laboratory (LNLS)/ Brazilian Center for Research in Energy and Materials (CNPEM), Campinas- Brazil.

2. Vegetal Biology Department of Biology Institute (IB)/ University of Campinas (UNICAMP), Campinas- Brazil.

3. Swiss Light Source (SLS)/ Paul Scherrer Institut (PSI), Villigen- Switzerland.

* Corresponding author, carla.polo@lnls.br

During plant development, lignin is deposited along with cellulose and hemicellulose in the secondary cell walls of structural fibers and water-conducting cells, being essential for their strength and rigidity [1]. Attempts to reduce the biomass recalcitrance through genetic manipulation of lignin deposition have met with some success, but the stunted growth of many of the resulting plants and the associated yield penalty have made the use of similar genetic modifications in commercial biomass crops problematic [2]. Although extensive biochemical characterization has already been performed showing chemical cell wall differences among wild-type and lignin-deficient mutants, information regarding the 3D structural changes introduced in the lignocellulosic layer is still missing. Ptychographic X-ray computed tomography (PXCT) is a coherent X-ray based technique, which, combined with tomography, enables to obtain a 3D density map of several tens of micrometers thick specimens with few tens of nanometers resolution [3]. Therefore, we present the use of PXCT to study the three-dimensional organization and interaction of the complex cross-linked molecules within the plant cell at the nanometer scale.

Wild type and $\mathrm{C} 4 \mathrm{H}$ Arabidopsis thaliana petiole fragments were selected for these studies and manually cut down to volumes of around $0.5 \times 0.5 \times 1 \mathrm{~mm}$. These fragments were chemically fixed and infiltrated with LR white acrylic resin. The resin block containing the small samples volume (Fig. 1A and 1B) were reduced to $50 \times 50 \mu \mathrm{m}(\mathrm{d} \times \mathrm{h}$ ) pillar (Fig. 1C) by Ga FIB-SEM (FEI Helios 600i) from the Scientific Center for Optical and Electron Microscopy (ScopeM-ETH Zurich). The pillars were transferred onto a flat $\mathrm{Au}$ coated pin and glued with platinum deposition (Fig. 1D). The PXCT measurements were done at cSAXS beamline of the Swiss Light Source (PSI- Villigen, Switzerland). The pin was introduced into the OMNY chamber enabling sample cryocooling [4] in order to reduce $\mathrm{X}$-ray radiation damage. The sample was placed $3 \mathrm{~mm}$ downstream the Fresnel zone plate $(220 \mu \mathrm{m}$ diameter with outermost width of $60 \mathrm{~nm}$, with a focal distance of $66 \mathrm{~mm}$ ) resulting in a $10 \times 10 \mu \mathrm{m}^{2}$ coherent X-ray beam, at $6.2 \mathrm{keV}$, with $10^{8}$ photons/s. The Eiger $500 \mathrm{~K}$ detector, with $75 \times 75 \mu \mathrm{m}^{2}$ pixel size was placed $7.3 \mathrm{~m}$ from the sample position. Ptychographic scans were performed with a circular pattern [3] with a step size of $2.5 \mu \mathrm{m}$ over a field of view of $60 \times 25 \mu \mathrm{m}^{2}$ (w x h) with a total number of 238 points/projection. Each point was exposed for $0.1 \mathrm{~s}$ and total angular coverage was $0^{\circ}$ to $180^{\circ}$ with an angular step of $0.48^{\circ}$ resulting in 750 projections. 


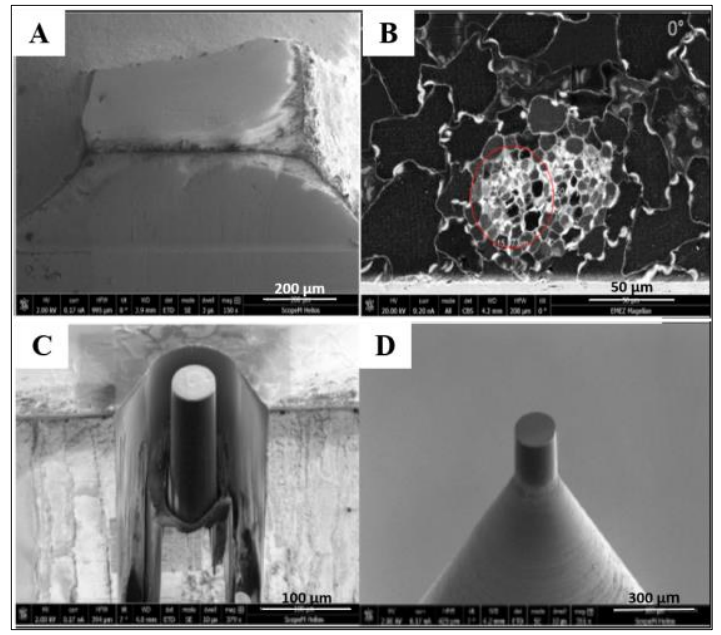

Figure 1. Sample preparation by GaFIBSEM. (A) Sample within resin block. (B) ROI localization into the block surface. (C) Ga beam shaped pillar containing the ROI. The $50 \times 50 \mu \mathrm{m}$ pillar transferred by the micromanupulator to the flat $\mathrm{Au}$ coated OMNY pins (D).

For each ptychographic projection, 2D diffraction patterns were recorded and used in the iterative phase retrieval algorithm to recover the $2 \mathrm{D}$ complex-valued transmissivity function of the specimen at each angular position. The reconstructions were performed using a maximum likelihood refinement [5]. Phase projections were further processed to remove a phase off-set and a linear phase term and alignment in $x$ and $y$ directions, with subpixel precision [6]. The refinement alignment in $x$ based on tomographic consistency was further applied [8]. Tomographic reconstructions were performed from wrapped phase projections by a modified filtered back projection (FBP) algorithm [7]. The 3D images were successfully reconstructed with $130 \mathrm{~nm}$-resolution and $48.87 \mathrm{~nm}$ voxel size. Figure 2 presents the longitudinal slices of the reconstructed tomogram for $A$.

thaliana wild type (A) and C4H (B) mutant. Preliminary image analysis allow identifying differences in lignin deposition in secondary cell walls observed in the $\mathrm{C} 4 \mathrm{H}$ mutant as compared to wild-type plant. The lignin content will be further quantified by image segmentation. Moreover, it is possible to observe the changes in cell morphology, since the mutant plant displays shorter and wider cells compared to the wild-type cells. We hypothesize that these morphological dissimilarities could be influenced by the lignin deposition patterns and could explain why mutant plants possess phenotypes such as dwarfism. The methodological approach for sample preparation and PXCT measurements at cryogenic

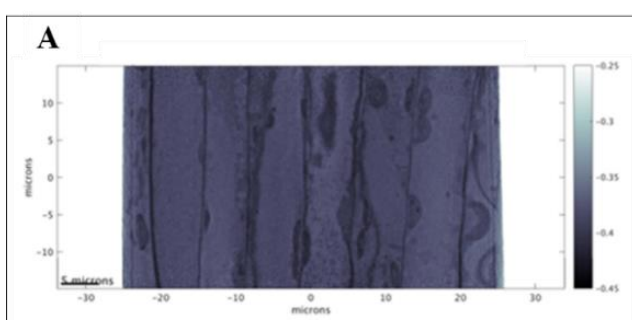

B

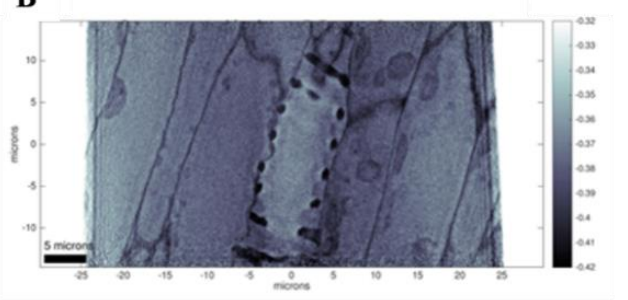

Figure 2. Transverse slice from PXCT reconstructed tomogram. (A) represents the wild type plant whereas (B), the $\mathrm{CH} 4$ mutant. conditions paves the way to access the 3D nanometric components in plants cells within their native vegetal tissue, and therefore, understand their role in plant physiology.

\section{References:}

[1] C Somerville et al, Science 306 (2004), p. 2206-11.

[2] S R Turner et al, Plant Cell 9 (1997), p.689-701.

[3] M Dierolf et al, Nature 467 (2010), p. 436-440.

[4] S H Shahmoradian et al, Scientific Reports 7 (2017) 6291.

[5] P Thibault and M Guizar-Sicairos, New Journal of Physics. 14 (2012) 063004.

[6] M Guizar-Sicairos et al, Optics Express 19 (2011), p. 21345-21357.

[7] M Holler et al, Scientific Reports 4 (2014) 3857.

[8] M Guizar-Sicairos et al, Optica 2 (2015), p. 259-266.

[9] The authors acknowledge funding from Brazilian Center for Research in Energy and Materials (CNPEM). L. Pereira and D. Flores are thanked for sample preparation and M. Guizar-Sicairos and M. Holler for discussions and contributions to this work. 